\title{
CASOS CONFIRMADOS DE MALÁRIA NO BRASIL ENTRE OS ANOS DE 2011 E 2015
}

\section{ARTIGO ORIGINAL}

ALMEIDA, Hellen Karine Santos ${ }^{1}$

FECURY, Amanda Alves ${ }^{2}$

OLIVEIRA, Euzébio ${ }^{3}$

DENDASCK, Carla Viana ${ }^{4}$

DIAS, Claudio Alberto Gellis de Mattos ${ }^{5}$

ALMEIDA, Hellen Karine Santos. Et al. Casos confirmados de malária no Brasil entre os anos de 2011 e 2015. Revista Científica Multidisciplinar Núcleo do Conhecimento. Ano 05, Ed. 04, Vol. 07, pp. 05-16. Abril de 2020. ISSN: 2448-0959, Link de acesso: https://www.nucleodoconhecimento.com.br/saude/malaria-no-brasil, DOI: 10.32749/nucleodoconhecimento.com.br/saude/malaria-no-brasil

1 Técnico em mineração pelo Instituto de Ensino Básico, Técnico e tecnológico do Amapá (IFAP).

${ }^{2}$ Biomédica, Doutora em Doenças Topicais, Professora e pesquisadora do Curso de Medicina do Campus Macapá, Universidade Federal do Amapá (UNIFAP).

3 Biólogo, Doutor em Doenças Topicais, Professor e pesquisador do Curso de Educação Física da, Universidade Federal do Pará (UFPA).

4 Teóloga, Doutora em Psicanálise, pesquisadora do Centro de Pesquisa e Estudos Avançados- CEPA.

${ }^{5}$ Biólogo, Doutor em Teoria e Pesquisa do Comportamento, Professor e pesquisador do Curso de Licenciatura em Química do Instituto de Ensino Básico, Técnico e tecnológico do Amapá (IFAP). 


\section{RESUMO}

A malária é uma doença mundial que causa um número elevado de mortes. Ela é causada pela picada do mosquito Anopholes infectado pelo protozoário parasita do gênero Plasmodium $O$ objetivo deste artigo é mostrar os números de casos confirmados de malária no Brasil, quanto os anos de confirmação, a faixa etária e região de notificação, entre os anos de 2011 a 2015. Dados retirados do departamento de informática do SUS, o DATASUS e de artigos. Houve um declínio do número do período citado, pessoas entre a faixa etária de 20 a 39 anos, seguido pela faixa entre 49 e 59 anos, o maior número de casos ocorreu com pessoas do sexo masculino, o maior número de casos ocorreu com pessoas de raça branca seguida por pardos, o maior número de casos por escolaridade é desconhecida onde a escolaridade é conhecida, os maiores números são com pessoas do ensino médio completo, seguido de pessoas com educação superior completa, maior número de casos ocorreu em área de zona urbana, a região sudeste possui o maior número de casos confirmados de malária no período, o maior número de casos ocorreram no estado de Rondônia. Conclui-se que campanhas tiveram influência na sociedade e colaboraram com a diminuição do número de doenças transmissíveis como a malária. Homens trabalham em áreas de maior risco de contaminação e em locais de proliferação do vetor, por isso estão mais expostos a áreas de proliferação do mosquito e ao contágio. No Brasil a maioria da população se reconhece como sendo branca e parda, respectivamente por isso os números mostram essas etnias como as mais infectadas. O mosquito parece se proliferar mais facilmente em lugares nos quais houve modificações causadas pelo homem. O meio urbano, por ser um local extremamente modificado, faz com que haja um maior número de casos devido à disponibilidade maior de criadouros. Acredita-se que por isso a região sudeste apresenta um maior número de casos e por ser, também, uma das regiões do país que mais sofreu modificações pelo homem. A abertura de rodovias e o aumento de assentamentos facilitam o contato do mosquito com o homem. O estado de Rondônia existe um grande número de assentamentos e desmatamentos para acomodar o progresso.

Palavras-chaves: Malária, epidemiologia, casos confirmados. 


\section{INTRODUÇÃO}

A malária é uma doença mundial que causa um número elevado de mortes (Aregawi et al,. 2017; Jotta e Carneiro, 2009; Camargo, 1995).

Ela é causada pela picada do mosquito Anopholes infectado pelo protozoário parasita do gênero Plasmodium. Pode ser adquirida por dois meios: o meio modificado pelo homem e pelo meio natural. O mais propício à transmissão é o meio urbano. A ação do homem transformando o ambiente parece fazer com que o mosquito causador da malária venha a proliferar e transmitir a doença (Barata, et al., 1993; Castro e Singer., 2007).

A malária possui diversos sintomas facilmente reconhecíveis. O principal deles e característico da doença é a febre alta. Há também o aumento do baço, acompanhado de dores de cabeça, náuseas, calafrios e mal-estar (Camargo, 1995; Aregawi et al,. 2017).

Antes do início do tratamento é necessário que haja conhecimento sobre a idade do paciente, a espécie do Plasmodium, gravidade da doença e as condições que o paciente se encontra (gravidez ou outros problemas de saúde). O tratamento se inicia com a interrupção da reprodução do parasita no organismo, utilizando drogas que impedem o desenvolvimento da doença. O Ministério da Saúde disponibiliza gratuitamente antimaláricos para todo o país. O tratamento mais aceito hoje, embora tenha novas técnicas, é o "exame de gota", um exame simples, de baixo custo e eficaz (Brasil, 2010; Brasil, 2017).

Para a prevenção da malária é fundamental evitar locais que possuam criadouros de malária, tais como rios e áreas alagadas. O uso de repelentes no corpo e telas nas portas e janelas é importante para pessoas que moram próximas as áreas de maior risco de contaminação da doença. (Brasil, 2015; Bentes, et al., 2017).

A malária está presente em cerca de 104 países ao redor do planeta. Estima-se a existência de cerca de 219 milhões de casos em 17 países. A maioria se encontra no 
continente africano, no qual se destaca a Nigéria e República Democrática do Congo. A América do Sul e América Central vem logo em seguida com $71 \%$ do úmero de casos. O sul e sudeste da Ásia fica com o menor número de casos registrados, 50\% no total. Na Europa e na América do Norte o número de casos é desconhecido ou nulo (Camargo, 2003; Santos, 2016).

O Brasil, embora tenha obtido uma diminuição no número de indivíduos infectados, ainda concentra um número muito elevado de casos confirmados de malária, cerca de 200 mil por ano, desde 2011. A região Norte do país apresentou 930.486 casos de malária entre 2011 e 2015 (Santos, 2016; Grillo, et al., 2017).

No Amapá o número de casos girou em torno de 13.817, divididos em assentamentos (655 casos), em garimpos ( 1.724 casos), em áreas indígenas (1490 casos), em áreas urbanas (2.410 casos) e em áreas rurais (7.538 casos) (Brasil, 2015).

\section{OBJETIVO}

O objetivo deste artigo é mostrar os números de casos confirmados de malária no Brasil, quanto os anos de confirmação, a faixa etária e região de notificação, entre os anos de 2011 a 2015.

\section{MÉTODO}

Dados retirados do departamento de informática do SUS, o DATASUS (http://datasus.saude.gov.br) seguindo as seguintes etapas: primeiro selecionou-se a aba "acesso à informação" em seguida a opção "informações de saúde (TABNET)" logo depois clicou-se em "epidemiológicas e morbidade". Na próxima página acessouse a opção "Doenças e Agravos de Notificação - De 2007 em diante (SINAM)". Na nova página selecionou-se o ícone "Malária". Na aba "abrangência geográfica" selecionou-se a opção "Brasil por Região, UF e Município". Para a coleta de dados seguiu-se os passos: selecionou-se no campo linha as opções "Ano $1^{\circ}$ sintomas", "faixa etária", "sexo", e "Região de residência", "zona de residência", "escolaridade", "raça" e "estados da região norte do Brasil". Para todas as opções acima foram 
selecionados no campo coluna a opção "não ativa"; no campo conteúdo a opção "casos confirmados"; e em períodos disponíveis dados de 2011 a 2015. A compilação dos dados foi feita dentro do aplicativo Excel, componente do pacote Office da Microsoft Corporation. A pesquisa bibliográfica foi 'realizada em artigos científicos, utilizando-se para busca computadores do laboratório de informática do Instituto Federal de Educação, Ciência e Tecnologia do Amapá, Campus Macapá, situado na: Rodovia BR 210 KM 3, s/n - Bairro Brasil Novo. CEP: 68.909-398, Macapá, Amapá, Brasil.

\section{RESULTADOS}

A figura 1 mostra a porcentagem de casos confirmados de malária no Brasil entre os anos de 2011 a 2015. Os dados mostram que houve um declínio do número do período citado.

Figura 1 Mostra a porcentagem de casos confirmados de malária no Brasil entre os anos de 2011 a 2015.

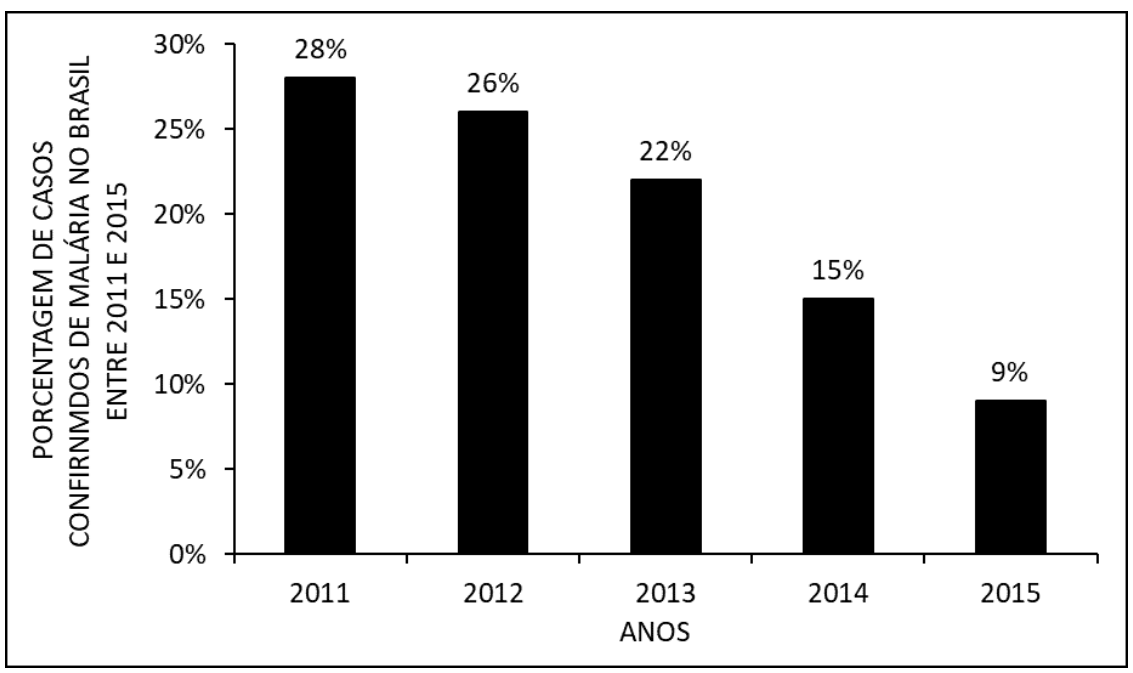

A figura 2 mostra a porcentagem de casos confirmados de malária no Brasil entre os anos de 2011 a 2015 por faixa etária. Os dados mostram que o maior número de casos de malária ocorreu com pessoas entre a faixa etária de 20 a 39 anos, seguido pela faixa entre 49 e 59 anos. 
Figura 2 Mostra a porcentagem de casos confirmados de malária no Brasil entre os anos de 2011 a 2015, por faixa etária.

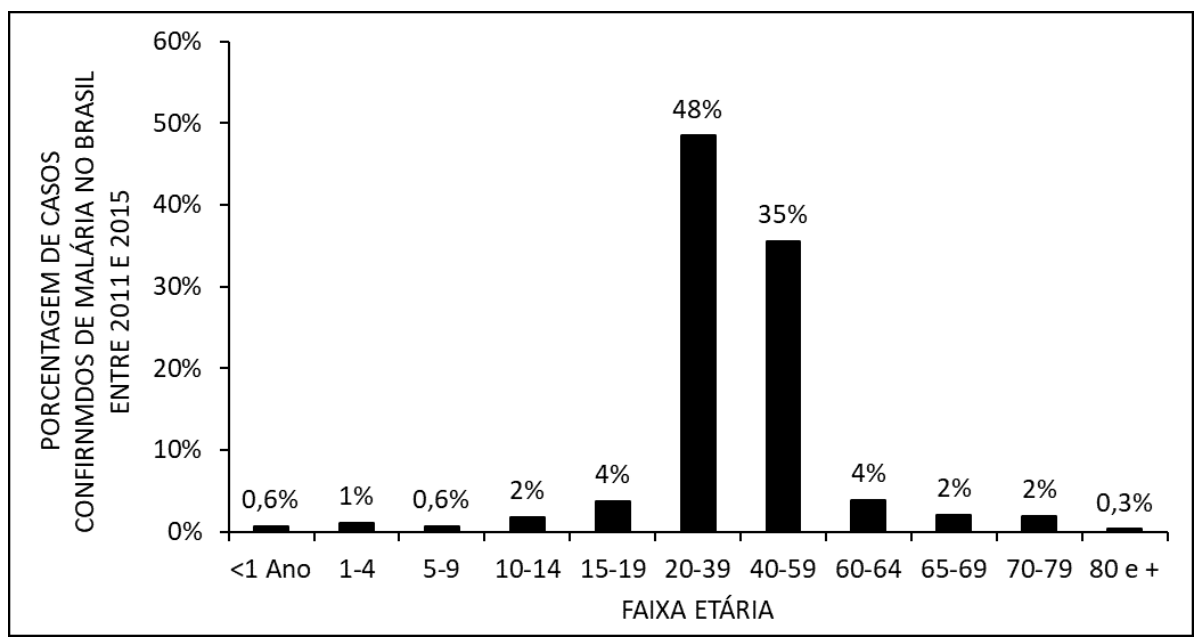

A figura 3 mostra a porcentagem de casos confirmados de malária no Brasil entre os anos de 2011 a 2015 por gênero. Os dados mostram que o maior número de casos ocorreu com pessoas do sexo masculino.

Figura 3 Mostra a porcentagem de casos confirmados de malária no Brasil entre os anos de 2011 a 2015 por gênero.

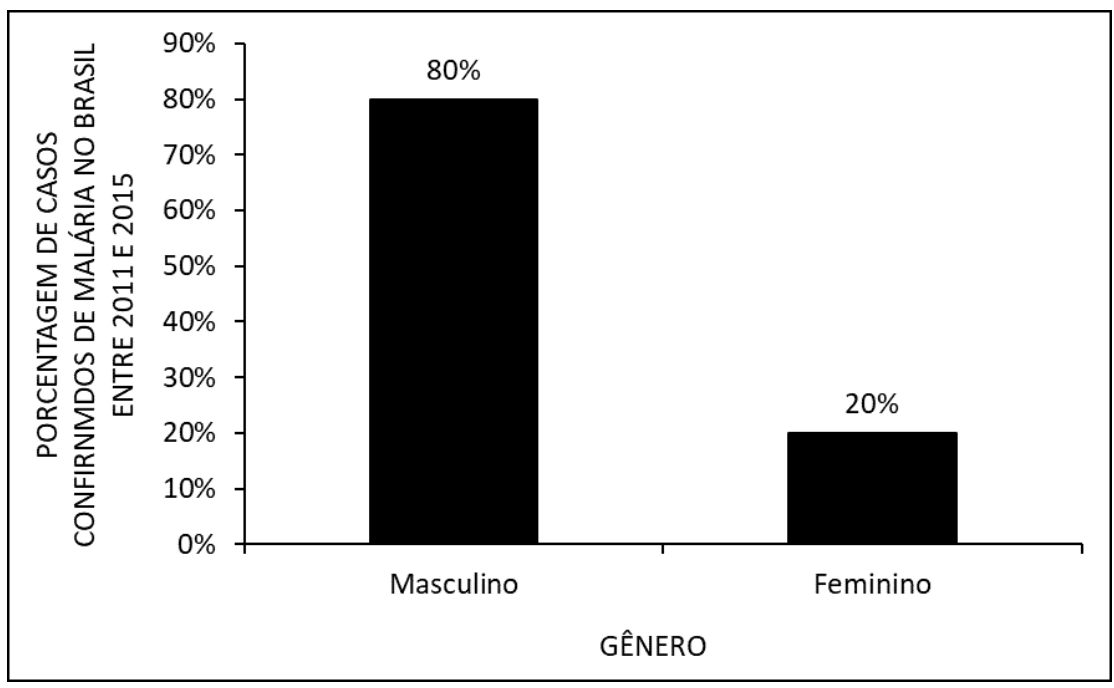

A figura 4 mostra a porcentagem de casos confirmados de malária no Brasil entre os anos de 2011 a 2015 por raça. Os dados mostram que a maior quantidade de casos ocorreu com pessoas de raça branca seguida por pardos. 
Figura 4 Mostra a porcentagem de casos confirmados de malária no Brasil entre os anos de 2011 a 2015 por raça.

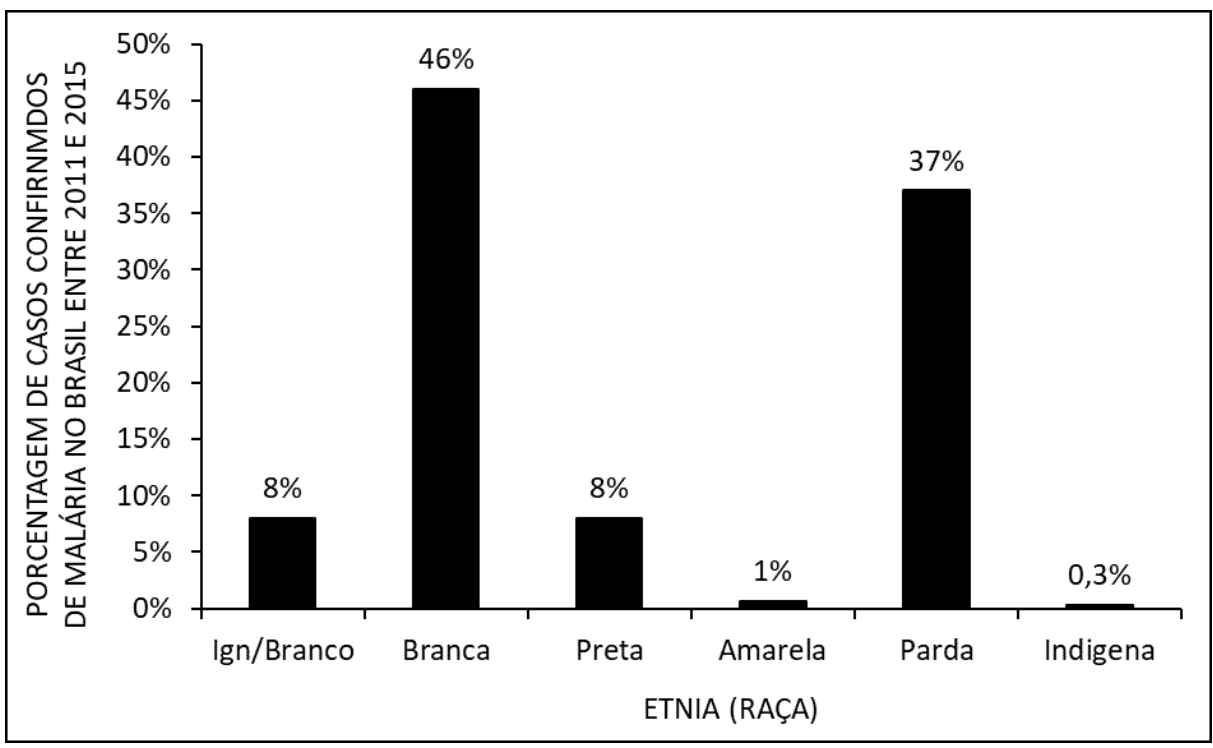

A figura 5 mostra a porcentagem de casos confirmados de malária no Brasil entre os anos de 2011 a 2015 por escolaridade. Os dados mostram que o maior número de casos por escolaridade é desconhecido. Onde a escolaridade é conhecida, os maiores números são com pessoas do ensino médio completo, seguido de pessoas com educação superior completa.

Figura 5 Mostra a porcentagem de casos confirmados de malária no Brasil entre os anos de 2011 a 2015 por escolaridade.

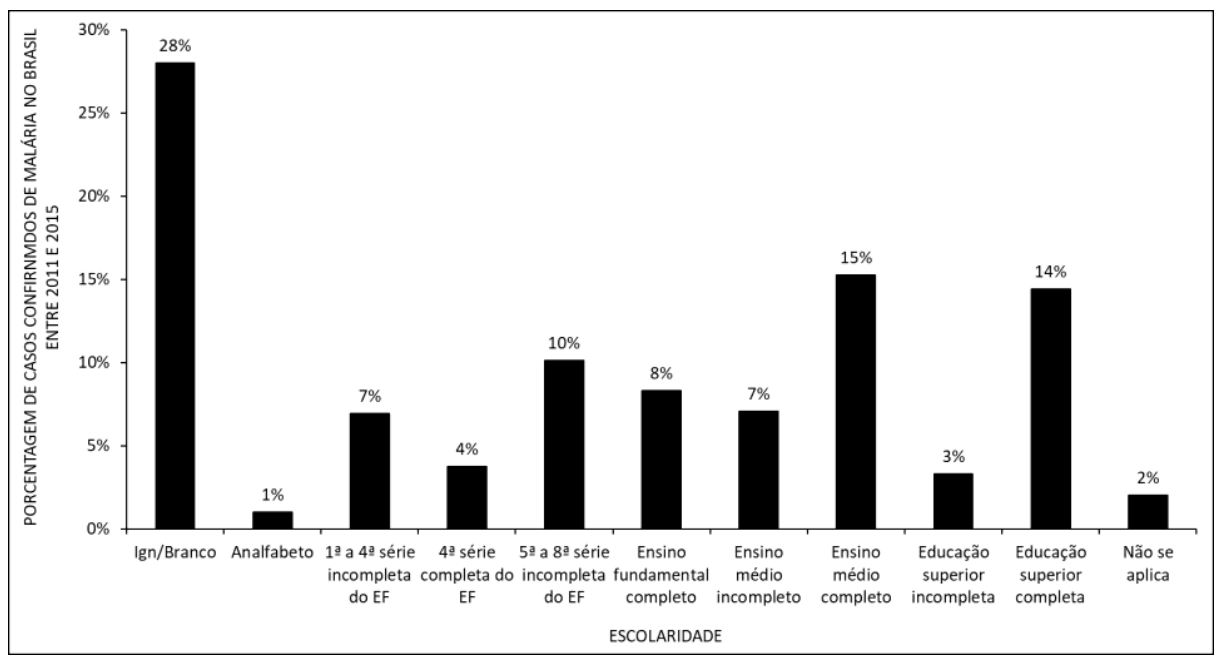


A figura 6 mostra a porcentagem de casos confirmados de malária no Brasil entre os anos de 2011 a 2015 por zona de residência. Os dados mostram que a maior quantidade de casos ocorreu em área de zona urbana.

Figura 6 Mostra a porcentagem de casos confirmados de malária no Brasil entre os anos de 2011 a 2015 por zona de residência.

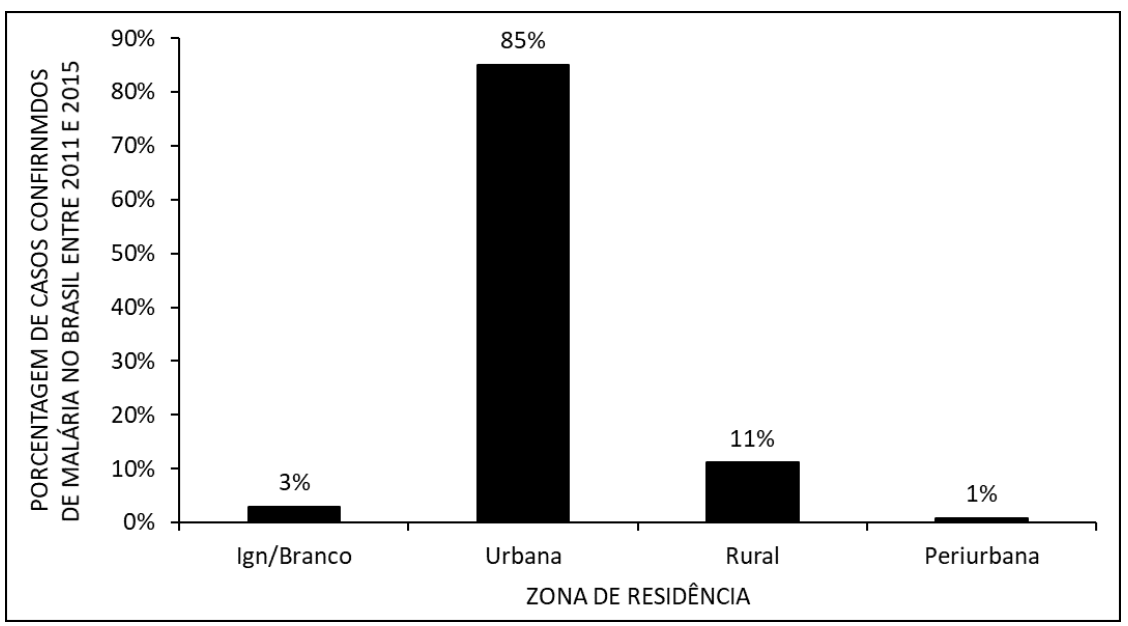

A figura 7 mostra a porcentagem de casos confirmados de malária no Brasil entre os anos de 2011 a 2015 por regiões do país. Os dados mostram que a região sudeste possui o maior número de casos confirmados de malária no período.

Figura 7 Mostra a porcentagem de casos confirmados de malária no Brasil entre os anos de 2011 a 2015 por regiões do país

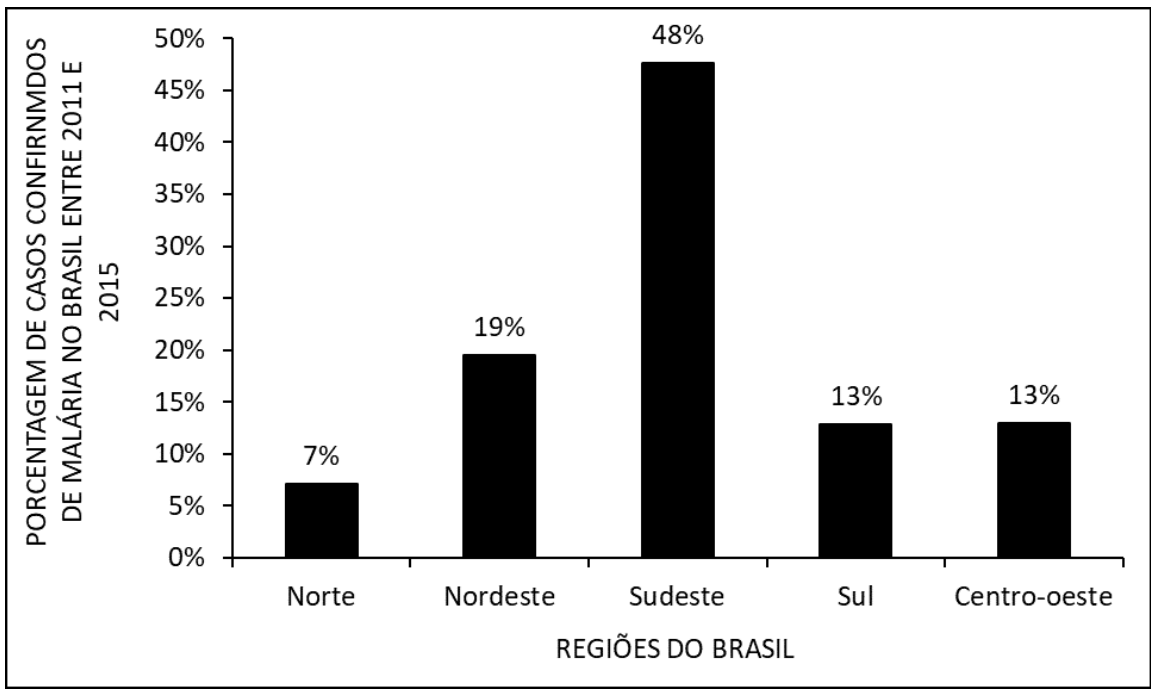

RC: 49044

Disponível em: https://www.nucleodoconhecimento.com.br/saude/malaria-no-brasil 
A figura 8 mostra a porcentagem de casos confirmados de malária no Brasil entre os anos de 2011 a 2015 por estados da região norte do Brasil. Os dados mostram que o maior número de casos ocorreu no estado de Rondônia.

Figura 8 Mostra a porcentagem de casos confirmados de malária no Brasil entre os anos de 2011 a 2015 por estados da região norte do Brasil.

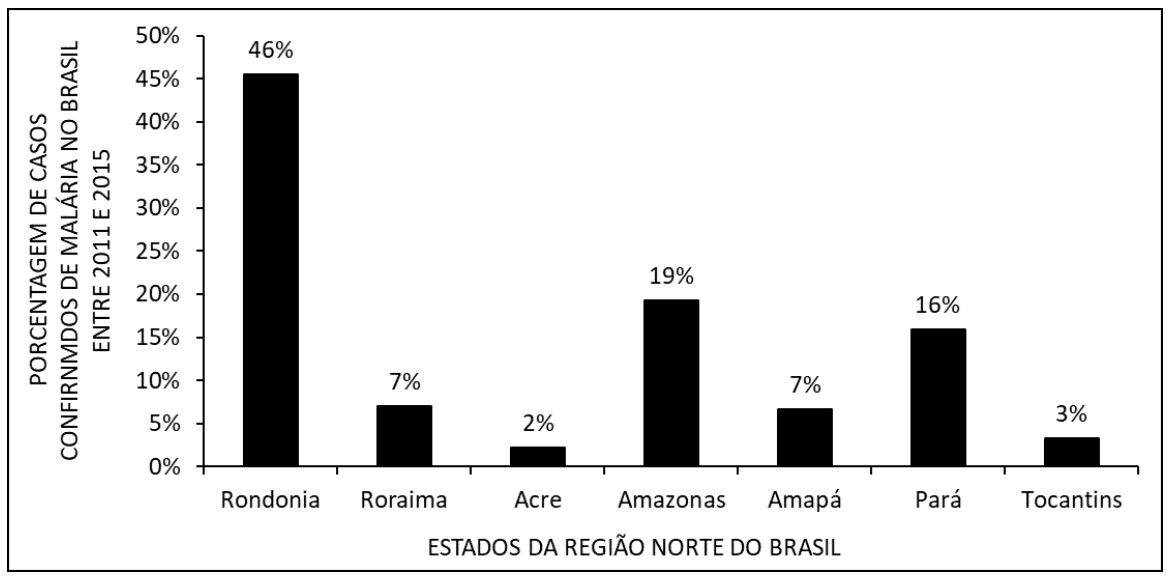

\section{DISCUSSÃO}

Os dados sobre o declínio no número de casos de malária entre os anos e 2011 a 2015 (figura 1) estão de acordo com a literatura. O Governo Federal ao analisar o grande número de casos de malária, realizou em 2015 a campanha "Elimine a Malária para o Bem". Esta campanha possivelmente teve grande influência na sociedade, com o número de casos apresentando uma redução de $89 \%$ comparado aos anos anteriores (BRASIL, 2017).

Os dados mostram que o maior número de casos de malária ocorreu com pessoas entre a faixa etária de 20 a 39 anos (figura 2). Parece que essa faixa etária é onde se encontra a maioria dos trabalhadores do país. As pessoas na sua grande maioria homens, trabalham em áreas de maior risco de contaminação e em locais de proliferação do vetor (JUNIOR e LEVY, 2014; LUPPI et al., 2014).

Os dados sobre o número de casos de malária por gênero mostram que o maior número de casos ocorreu com pessoas do sexo masculino (figura 3). As mulheres 
parecem se preocupar mais com a saúde e com isso previnem muito mais as doenças. Quanto que os homens procuram somente quando a doença já está instalada (PINHEIRO et al., 2002). Os homens também estão mais propícios a trabalhar em áreas de proliferação do mosquito (LUPPI et al., 2014).

A figura 4 mostra que o maior número de casos ocorreu com pessoas de etnia branca seguido por pardos. No Brasil a etnia é autodeclarada e a maioria da população se reconhece como sendo branca e parda, respectivamente (PEREIRA et al., 2014).

Os dados mostram que o maior número de casos de malária no Brasil ocorre com pessoas do ensino médio completo, seguido de pessoas com educação superior completa (figura 5). Segundo o IBGE (2017), houve um aumento no grau de escolaridade do brasileiro entre 2007 a 2014. Outro provável motivo é a instalação de indústrias em locais onde há maior proliferação do mosquito. A demanda dessas empresas é por profissionais que tenham uma boa qualificação, logo, pessoas que tenham ensino médio e superior completo (LUPPI et al., 2014).

A figura 6 mostra que o maior número de casos ocorreu em área de zona urbana. $\mathrm{O}$ mosquito parece se proliferar mais facilmente em lugares nos quais houve modificações causadas pelo homem. O meio urbano, por ser um local extremamente modificado, faz com que haja um maior número de casos devido à disponibilidade maior de criadouros (BARATA, et al., 1993.; VIEIRA et al., 2014).

A região sudeste do Brasil possui o maior número de casos confirmados de malária no período estudado (figura 7). Esta é uma das maiores e a mais desenvolvida região do país, apresentando um maior número de pessoas em detrimento das outras regiões. Acredita-se que por isso esta região apresenta um maior número de casos e por ser, também, uma das regiões do país que mais sofreu modificações pelo homem, o que parece facilitar a proliferação da malária (GUIMARÃES et al., 2015; BARATA, et al., 1993).

Dos estados da região norte do Brasil Rondônia é o que parece apresentar o maior número de casos de malária (figura 8). Parece que a abertura de rodovias e o aumento 
de assentamentos facilitam o contato do mosquito com o homem. O estado de Rondônia existe um grande número de assentamentos e de desmatamentos para acomodar o progresso (VIEIRA et al., 2014; BRASIL, 2015).

\section{CONCLUSÃO}

Campanhas tiveram influência na sociedade e colaboraram com a diminuição do número de doenças transmissíveis como a malária.

Homens trabalham em áreas de maior risco de contaminação e em locais de proliferação do vetor, por isso estão mais expostos a áreas de proliferação do mosquito e ao contágio.

No Brasil a maioria da população se reconhece como sendo branca e parda, respectivamente por isso os números mostram essas etnias como as mais infectadas.

O mosquito parece se proliferar mais facilmente em lugares nos quais houve modificações causadas pelo homem. O meio urbano, por ser um local extremamente modificado, faz com que haja um maior número de casos devido à disponibilidade maior de criadouros. Acredita-se que por isso a região sudeste apresenta um maior número de casos e por ser, também, uma das regiões do país que mais sofreu modificações pelo homem.

A abertura de rodovias e o aumento de assentamentos facilitam o contato do mosquito com o homem. O estado de Rondônia existe um grande número de assentamentos e desmatamentos para acomodar o progresso.

\section{REFERÊNCIAS}

AREGAWI, S.; LI, L.; MIRAGLIA, C.M. Malaria rapid diagnostic test and Giemsa stained peripheral blood smear discrepancies in the diagnosis of Plasmodium ovale infection in New England. CLINICAL LABORATORY SCIENCE, v.30,2017. 
BARATA, L. C. B.; ANDRIGUETTI, M. T. M. MATTOS, M. R. Surto de malária induzida entre usuários de drogas injetáveis. Rev. Saúde Pública, 27(1), 1993.

BRASIL. Ministério da Saúde. Secretaria de Vigilância em Saúde. Departamento de Vigilância Epidemiológica. Guia prático de tratamento da malária no Brasil / Ministério da Saúde, Secretaria de Vigilância em Saúde, Departamento de Vigilância Epidemiológica. - Brasília : Ministério da Saúde, 2010. 36 p. : il. color. - (Série A. Normas e Manuais Técnicos).

BRASIL. Ministério da Saúde. Secretaria de Vigilância em Saúde. Departamento de Vigilância Epidemiológica. Boletim Epidemiológico/ Ministério da Saúde, Secretaria de Vigilância em Saúde, Departamento de Vigilância Epidemiológica. - Brasília : Ministério da Saúde, V.46 N. 43, 2015

BRASIL, Brasil registra menor número de casos de malária nos últimos 35 anos. Disponível em: www.portalsaude.saude.gov.br. Acesso em: 19, setembro de 2017.

CAMARGO, E. P. Malária, Maleita, Paludismo. Ciência e Cultura, vol.55 no.1 São Paulo Jan./Mar 2003.

CAMARGO, E. P. A malária encenada no grande teatro social. Estudos Avançados, $9(24), 1995$.

CASTRO, M. C.; SINGER, B. H. Meio ambiente e saúde: metodologia para análise espacial da ocorrência de malária em projetos de assentamento. R. bras. Est. Pop., São Paulo, v. 24, n. 2, p. 247-262, jul./dez. 2007

GRILLO, M. L.; SILVA, L. T.; ARAÚJO, A. G. J; FARIAS, J. F. S.; RODRIGUEZ, D. A.; ROFATTO, F. A.; PRADO, M. L.; SILVA, L. G. B. A. Evolução do Número de Pessoas Infectadas por Malária no Brasil entre 2003 e 2015. I Encontro Acadêmico da Engenharia Ambiental da ELL-USP, 2017.

GUIMARÃES, R. M.; ANDRADE, S. S. C. A.; MACHADO, E. L.; BAHIA, C. A.; OLIVEIRA, M. M.; JACQUES, F. V. L. Diferenças regionais na transição da 
mortalidade por doenças cardiovasculares no Brasil, 1980 a 2012. Rev Panam Salud Publica. 2015;37(2):83-9.

IBGE. Governo Federal. Educação. Disponivel em: $<$ https://brasilemsintese.ibge.gov.br/educacao.html>. Acessado no dia 03 de outubro de 2017.

JOTTA, L. A. C. V.; CARNEIRO, M. H. S. Malária: As Imagens Utilizadas em Livros Didáticos de Biologia. Encontro Nacional de Pesquisa em Educação em Ciência. $8 \mathrm{nov} / 2009$.

JÚNIOR, J. R. C. S.; LEVY, P. M. Impactos do Declínio do Bônus Demográfico Sobre a Oferta de Mão de Obra e o Crescimento Econômico no Período de 2014-2030. Carta de Conjuntura, 25/ dez. 2014.

LUPPI, O.; VIDIGAL, A. C.; LONGO, C.; COSTA, A. P.; SARAIVA, R. P.; RIBEIRO, C. T. D.; BRASIL, P. Estudo dos casos suspeitos de malária importada, um Centro de Referência na região extra-Amazônica. Centro de Informação Estratégica em Vigilância em Saúde, FIOCRUZ; Instituto de Pesquisa Clínica Evandro Chagas, FIOCRUZ, Brasil, 2014.

PINHEIRO, R. S.; VIACAVA, F.; TRAVASSOS, C.; BRITO, A. S. Gênero, morbidade, acesso e utilização de serviços de saúde no Brasil. Ciência \& Saúde Coletiva, 7(4):687-707, 2002.

SANTOS, J. A. G. Adesão ao tratamento da malária vivax em crianças. 2016. 74 f. Dissertação (Mestrado) - Universidade Federal do Pará, Instituto de Ciências da Saúde, Belém, 2016. Programa de Pós-Graduação em Ciências Farmacêuticas.

Enviado: Abril, 2020.

Aprovado: Abril, 2020. 